\title{
Analysis and Calibration of RDII and Design of Sewer Collection Systems
}

\author{
By Misgana K. Muleta and Paul F. Boulos
}

\begin{abstract}
Excessive wet weather flow resulting from rainfall-derived inflow and infiltration (RDII) is a major source of sanitary sewer overflows (SSOs). SSOs pose serious problem to the public and the environment by causing back up into basements and sewer overflows to streets and rivers. Control of sewer overflows is, therefore, vital to reducing risks to public health and protecting the environment from water pollution. Computer modeling of sewer collection systems plays an important role in determining sound and economical remedial solutions that reduce RDII, improve system integrity, reliability and performance, and avoid overflows. This paper presents a rigorous and efficient three-step optimization methodology for use in solving the sewer overflow problem. The first step analyzes measured sewer flow and rainfall data and decomposes the flow data into dryweather flow and wet-weather flow components. The second step computes the optimal RTK parameters of the tri-triangular unit hydrograph that is commonly used to model RDII into the sewer collection system. The optimal RTK parameters are calibrated with genetic algorithm so that the simulated RDII flows closely match the RDII time series generated by decomposing the measured flow data. In the final step, the calibrated model is then used with genetic algorithm to design cost-effective solutions for existing SSO problems. Design parameters can include any combinations of pipe size, storage, slope, and pumping. The proposed wet-weather flow decomposition, optimal calibration, and optimal design models are demonstrated using an example sewer collection system. The methodology seems a good alternative to other methods proposed in the literature and should prove useful for engineers and planners that are involved in mitigating complex SSO problems.
\end{abstract}

\section{Introduction}

Sanitary sewer collection systems are designed to collect and transport sanitary wastewater such as domestic sewage from homes, wastewaters from industrial and 
commercial facilities, and certain amounts of wet-weather flows to wastewater treatment plants without causing overflows. However, sanitary sewer overflows (SSOs) may occur when the capacity of the collection system is exceeded due to excess wet weather flows, system blockages, or when power and mechanical failures prevent the system from operating properly. SSOs release partially treated or untreated sewage to surface waters, streets and basements. This unintentionally released sewage may contain microbial pathogens, toxics, nutrients, suspended solids and other pollutants that can pose risk to the public and the environment. The Environmental Protection Agency (EPA) estimated that between 23,000 and 75,000 SSOs occur each year in the United States, resulting in releases of between 3 billion and 10 billion gallons of untreated wastewater (EPA, 2004). To control sewer overflows, the EPA under the authority of the Clean Water Act adopted by Congress has implemented pollution control programs and set wastewater standards for the industry.

Rainfall-derived infiltration and inflow (RDII) into a sanitary sewer is the main cause of SSOs and other operational problems for many sewer systems. Although sanitary sewer systems are generally designed to accommodate RDII flows, these flows often exceed the design allowances. RDII represents total wet weather flow that enters the collection system in the form of inflow and infiltration. Inflow is water that enters the sewer system directly via depressed manhole lids and frames, downspouts, sump pumps, foundation drains, area way drains and cross-connections with storm sewers. Inflow typically occurs shortly after a rainfall starts and recedes quickly once it stops and typically accounts for the major component of the RDII peak flow. Infiltration refers to runoff that infiltrates into the soil before entering a sanitary sewer system through damaged pipe sections, leaky joints or poor manhole connections. Infiltration processes typically extend beyond the end of rainfall and takes some time to recede to zero after the storm event. Both infiltration and inflow increase with age of the sanitary sewer infrastructure.

Mathematical drainage modeling can be used to analyze existing sewer collection systems, to identify potential problems, and to design optimal remedial solutions (Nicklow et al., 2004, 2006). For sanitary sewer systems in particular, the ability to determine RDII flows reliably is critical for developing SSO control plans. The processes that convert rainfall to RDII flow in sanitary sewer systems are very complicated. Various factors control RDII responses in addition to the rainfall and antecedent moisture conditions, including depth to groundwater, depth to bedrock, land slope, number and size of sewer system defects, type of storm drainage system, soil characteristics, and type of sewer backfill. Furthermore, RDII responses can vary greatly due to spatial rainfall distributions over a sewershed. The industry standard and most accurate RDII prediction method is the USEPA RTK synthetic unit hydrograph (Bennett, 1999; Rossman, 2005).

In this paper, a rigorous and efficient three-step optimization methodology is used for solving the sewer overflow problem. First, a comprehensive QA/QC of rainfall and flow data is performed and the flow data decomposed into distinct dry-weather flow (DWF) and wet-weather flow (RDII) components using criteria such as rainfall threshold. The DWF component is further analyzed to construct a DWF pattern that can be used to 
simulate the system using the industry standard EPA SWMM5 mathematical drainage model (Rossman, 2005). The DWF pattern is then assigned to the source nodes that contribute DWF to the meter location in proportion to sewershed areas or based on other user-defined criteria. The RDII component is then analyzed to determine RDII events and to calibrate parameters of the RTK synthetic unit hydrograph so that the RDII flow simulated by the RTK method (Rossman, 2005) closely matches the RDII flow obtained by the decomposition process. The RTK unit hydrograph parameters are calibrated with genetic algorithm optimization. The calibrated RTK parameters and the DWF patterns are then passed to SWMM5 to carry out detailed dynamic flow routing through the sewer system and evaluate system response to support development of an optimal capital improvement program. The optimal design problem is then solved with genetic algorithm. This three-step methodology has been implemented into InfoSWMM (Boulos, 2005) and $\mathrm{H}_{2} \mathrm{OMAP}$ SWMM (Boulos, 2006) comprehensive geospatial decision support systems.

For systems that are experiencing SSOs and other performance issues, design improvement alternatives commonly evaluated include the addition of new sewer pipes or treatment capacity, increasing conduit capacity (bigger interceptors), more storage volume, pumping capacity, and efficient utilization of existing storage in the system through implementation of real time operational controls. The daunting task is, however, to select an improvement option or a combination of options that effectively solves the SSO problem with the least capital expenditure. No single design improvement option can be generalized to every collection system. Performance and economic effectiveness of the remedial design alternatives will vary from system to system. The current practice of selecting an improvement option involves a tedious trial-and-error evaluation procedure that seldom leads to the most effective or most economical solution for upgrading collection systems.

The SWMM5 drainage network simulation model can be used to evaluate the hydraulic performance of the existing sewer system for different design alternatives (modifications) under a range of loading and operating conditions. The design option that meets the target hydraulic criteria with the lowest cost is then selected among the alternative designs. The complexity of this manual trial-and-error procedure increases exponentially with the number of proposed system modifications and corresponding operating conditions. It is important to point out that even if the target performance specifications are met, the trial-and-error procedure has no inherent feature that assures that the solution reached is cost optimum or even cost effective. Good engineering procedure dictates that the iterations continue until a number of promising alternatives have been evaluated. However, given the vast number of possible combinations of system enhancements, it is unlikely that even the most experienced modeler will be able to determine the least-cost improvement alternative using a trial-and-error procedure. Therefore, the result of using the traditional trial-and-error evaluation approach is often inefficient performance at a greater cost.

One way to circumvent the limitations of a trial-and-error procedure is to employ optimization theory. This paper presents an optimal design methodology for mitigating 
SSO problems in sewer systems. The method consists of determining the optimal design improvement solution that produces the minimum overall cost while satisfying target system performance requirements. The decision variables can include any selected combination of pipe slope and upsizing, storage, pumping and new piping. Performance criteria include maximum allowable depth to diameter ratio, minimum and maximum conduit velocities, maximum head loss for force mains, and minimum and maximum conduit slopes. This gives practicing engineers complete control over the solution process. The proposed approach links an extended version of the EPA SWMM5 drainage network simulator with a genetic algorithm-based optimization model and iterates between the simulator and the optimization model until optimal design solutions are found. The optimization model generates improved sets of decision variables that seek to minimize design costs and maximize system performance.

\section{Data Analysis and Flow Decomposition}

The wastewater flow monitoring data at sewer collection systems consists of dryweather flow components and RDII flow components. The dry-weather flow component can be further classified into ground water flow and base flow. Groundwater flow represents the groundwater infiltration that enters the collection system through defective pipes, pipe joints, and leaking manhole walls irrespective of rainfall availability. Base wastewater flow represents sewage from residential, commercial, and industrial areas released to the sanitary sewer system. RDII is the rainfall-driven flow that makes its way to the collection system. A crucial step towards successful modeling of sewer collection systems is the ability to decompose the flow monitoring data into base wastewater flow, ground water flow and the RDII flow. The decomposition process can then be used to understand the sources of flow and the relative quantities of each flow components for the sewer system. Additionally, it determines if RDII and groundwater flow components are excessive to cause SSOs and other operational problems.

Before decomposition is carried out, it is imperative to carefully analyze both rainfall data and flow data and to perform rigorous QA/QC. The analysis tool used in this study allows to review and edit data and to correct suspicious records. Missing data can be estimated by interpolation. Days with non-representative flow records (e.g., holidays) can be discarded from the analysis. Data affected by meter failure, system disruptions or other factors that could cause abnormal wastewater flows can also be removed. Once the flow data and the rainfall data are reviewed and corrected, the decomposition process begins by identifying dry days and wet days to determine the dry-weather flow components and the RDII components. A day is defined as dry if rainfall depth is less than a pre-specified threshold within a given duration. In addition, statistical analysis is employed to further test if a day qualifies as a dry day. Groundwater flow is determined based on the dry days. Next, groundwater flow is subtracted from flow data for the identified dry days to determine base wastewater flow (BWF). The BWF component is grouped into weekdays and weekend days and average hourly base wastewater flow values are determined for each group. The BWF pattern and the GWF pattern is allocated to the tributary nodes in proportion to the sewershed area and other user-defined criteria. 
RDII flow is then determined by subtracting the BWF pattern and the groundwater flow pattern from the total measured flow data.

\section{RDII Unit Hydrograph Calibration}

EPA SWMM 5 uses the RTK unit hydrograph (UH) to estimate RDII flow into a sewer system. A RTK UH set contains up to three such hydrographs, one for a short-term response, one for an intermediate-term response, and one for a long-term response. Each unit hydrograph, shown in Figure 1, is defined by three parameters. The $\mathrm{R}$ parameter represents the fraction of rainfall volume that enters the sewer system, $\mathrm{T}$ represents the time from the onset of rainfall to the peak of the UH in hours, and $\mathrm{K}$ represents the ratio of time to recession of the UH to the time to peak. A UH group can also have a set of Initial Abstraction (IA) parameters associated with it. These parameters determine how much rainfall is lost to interception and depression storage before any excess rainfall is generated and transformed into RDII flow by a unit hydrograph. The IA parameters consist of a maximum possible depth of IA (inches or $\mathrm{mm}$ ), a recovery rate (inches/day or $\mathrm{mm}$ /day) at which stored IA is depleted during dry periods, and an initial depth of stored IA (inches or mm). Accurate determination (calibration) of the UH parameters is crucial to simulate RDII flow using EPA SWMM 5 with the degree of certainty required to design sound mitigation plans to control SSOs and other operational problems

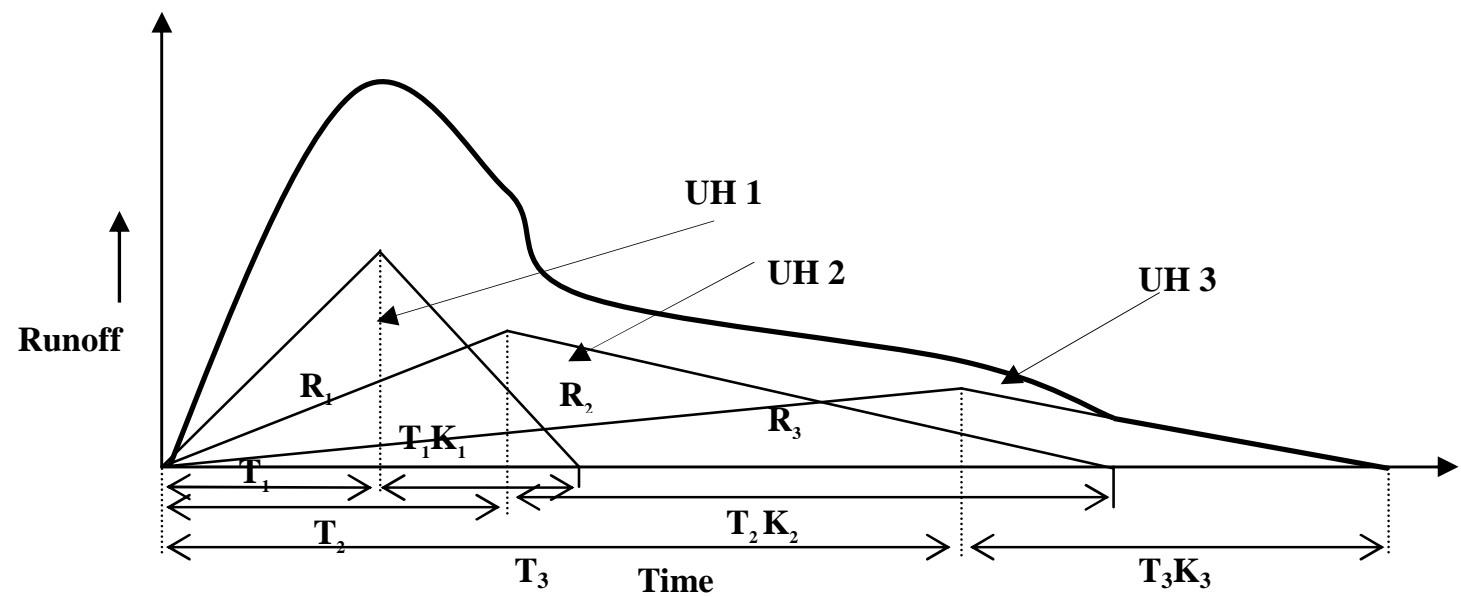

\section{Figure 1: RTK Unit Hydrograph}

Traditionally, calibration of RDII UH parameters is performed through a tedious and inexact trial-and-error process in which they are manually adjusted in an iterative fashion to closely match wet-weather flow data. Since there are a vast number of possible combinations of RTK values, evaluating all options this way may not be manageable, and even knowledgeable modelers often fail to obtain good results. In this paper, Genetic Algorithm (GA) optimization is used to automatically determine the UH parameters that best match the RDII time series generated by decomposing the measured flow data.

GA (Holland, 1975) starts the search process with a population of candidate solutions. Each of these solutions is made up of a certain value for every calibrable RDII 
parameter, where the values are randomly selected from the feasible range, defined in terms of the minimum and maximum bound specified for the individual parameters. Relative fitness of the candidate solutions is evaluated by comparing model simulations with the RDII flow determined by the decomposition process. Fitness evaluation is performed using the root mean square error. Fittest alternatives (i.e., solutions that bring model simulations closer to observed data) are given better chance to participate in creating new solution candidates. The process of determining relative fitness of solution candidates and creating new alternatives continues until the stopping criterion is satisfied. The search process is terminated when one or more of the following criteria is met: (1) fitness value exceeding a specified threshold (e.g., root mean square error less than 0.05); (2) reaching the maximum number of model simulations allowed; and (3) failing to achieve specified improvement in the fitness criterion within specified number of consecutive generations.

\section{Designing Remedial Solutions}

The most common solutions for mitigating sewer overflows and other operational problems include adding storage volume, increasing conduit capacity, expanding pumping capacity, and implementing real time operational controls to more effectively utilize existing system storage. Comprehensive modeling and analysis of these sewer systems becomes necessary for developing sound cost-effective and reliable solutions for enhancing system integrity and performance to convey sewer flows without causing overflows. However, identification of the optimal design solution that effectively circumvents overflow problems with the least expenditure is a daunting task. The current practice involves a tedious trial-and-error evaluation procedure that seldom leads to the most effective or most economical designs. An emerging design approach that is utilized in this work utilizes single objective optimization that identifies the solution that best satisfies a predefined criterion. The goal of the optimization formulation is to identify the "best" combination of conduit sizes, storage volumes, and pumping capacities needed for one or more facilities in the collection system that meet target system performance requirements (e.g., avoid flooding) at minimum cost. The sewer collection system optimization problem is thus governed by an objective function and its associated set of constraints. The objective (cost) function can be mathematically expressed as:

$$
\text { Minimize } \quad \sum_{c=1}^{C} C_{c} L_{c}+\sum_{s=1}^{S} C_{s} V_{s}+\sum_{p=1}^{P} C_{p}+\text { Penalty }
$$

where $c, C_{c}$ and $L_{c}$ used in the left-hand side term of the equation refer to the number of conduits, the replacement cost which is a function of conduit size, and the length of the conduit, respectively. Likewise the $s, C_{s}$, and $V_{s}$ used in the second term refer to storage node counter, cost per unit volume of storage and volume of the storage unit, respectively. The $p$ and $C_{p}$ used in the third term refer to pump counter and cost associated with the pump, respectively. The decision variables, which consist of conduit, storage and pump sizes, are automatically determined to minimize the objective function while satisfying the implicit system constraints, explicit bound constraints and explicit variable constraints. The implicit constraints on the sewer collection system are equality 
constraints defining the hydraulic equilibrium state of the system. They correspond to the conservation of mass and momentum equations (Saint Venant equations) that govern the unsteady flow of water through a drainage network of channels and pipes. These equations are solved implicitly using SWMM5, which computes the flow in each conduit and head at each node for each time step of the simulation period.

The explicit bound constraints represent system performance criteria and may include constraints on conduit flow velocity $(V)$, conduit slope $(S)$, and head loss $(H G)$ in force mains for a given set of sewer system loading and operating conditions. Conduit constraints describe hydraulic limits on the conduits. These limits include minimum and maximum velocities, maximum head losses for force mains, and maximum and minimum invert elevations. The minimum velocity constraint ensures that a sufficient velocity is maintained to keep solids in suspension and to encourage passage of sewer floatables. The maximum velocity criterion is critical for pressurized mains to ensure that momentum forces are not objectionably high on the system especially at joints. For gravity mains, maximum velocity is usually not a significant issue; however, under some conditions, the transition from supercritical to subcritical flows can create a large hydraulic jump and may be of concern to the engineer. The maximum head loss constraint ensures that pressurized mains have sufficient lift to carry the water to the upstream location.

A penalty cost is added to the objective function to penalize an infeasible solution (degrade its fitness) and force the search procedure towards the region of feasible solutions. The penalty cost function is defined as the divergence (distance) of the computed solution from the feasible region or:

$$
P C=\sum_{i=1}^{N} v_{i}\left|C_{i}(x)-C_{i}\right|
$$

where $N$ represents the number of constraints; $\mathrm{v}_{\mathrm{i}}$ represents a weighting factor associated to constraint $C_{i}, C_{i}(x)$ is the value of the $i^{\text {th }}$ constraint and $C_{\mathrm{i}}$ is the constraint limit. It is expected that different values of the penalty costs will result in different solutions and also affect the efficacy of the optimization calculation. Therefore, a number of trial optimization runs with different penalty costs may be required to better explore the solution space and narrow in to the lowest cost solutions.

The explicit variable constraints are used to set minimum (lower) and maximum (upper) limits on the sewer sizes and to specify the discrete (commercially available) diameter values for the new conduits. Conduits should be lumped together in separate logical design groups based on their known physical characteristics such as size and location. As such, all conduits within a group will possess an identical size. For each conduit group, the conduit size is bound by an explicit inequality constraint as:

$$
\operatorname{Dmin}_{n} \leq D_{n} \leq \operatorname{Dmax}_{n} \quad \forall n, \forall D_{n} \in D^{0}=\left\{d_{k}^{0}, k=1, \ldots K\right\}
$$


where $\operatorname{Dmin}_{n}$ designates the lower bound (the minimum value) of conduit sizes for conduit group $n$; $\operatorname{Dmax}_{n}$ represents the upper bound (the maximum value) of conduit sizes for conduit group $n$; and $D_{n}$ is the conduit size for conduit group $n$ and selected from a set of available conduit sizes of $D^{0}$. Similarly, pump curves are selected from various user-specified pump curves to represent pump station expansion. Storage volumes are computed based on shape of storage, defined either as a mathematical function or in tabular form (head vs. area), and are bound by user-specified minimum and maximum storage depths.

The optimal urban drainage system design/rehabilitation problem formulated above is solved using a dual-level optimal control methodology that integrates SWMM5 drainage network simulator with GA optimization. Starting with an initial feasible set of decision variables, it is passed to SWMM5 for use in explicitly satisfying the implicit system constraints and in evaluating the implicit bound constraints. The SWMM5 solution is then passed back to the optimization model for use in quantifying the objective function and any violations in the implicit bound constraints. This information is then utilized to produce an improved set of decision variables that automatically satisfies the explicit variable constraints and that seeks to minimize the objective function. This iterative process is repeated until the optimal solution is found. The resulting decision support optimization model (InfoSWMM and $\mathrm{H}_{2}$ OMAP SWMM) has been successfully applied to a number of actual sewer collection systems in the US. The results showed that significant improvements in system performance could be realized.

\section{Example Application}

The calibration model and the optimal design algorithm are demonstrated using an example sewer collection system. The RTK hydrograph was calibrated to the RDII flow determined by the decomposition process. All nine parameters of the RTK unit hydrograph were selected as decision variables for calibration.

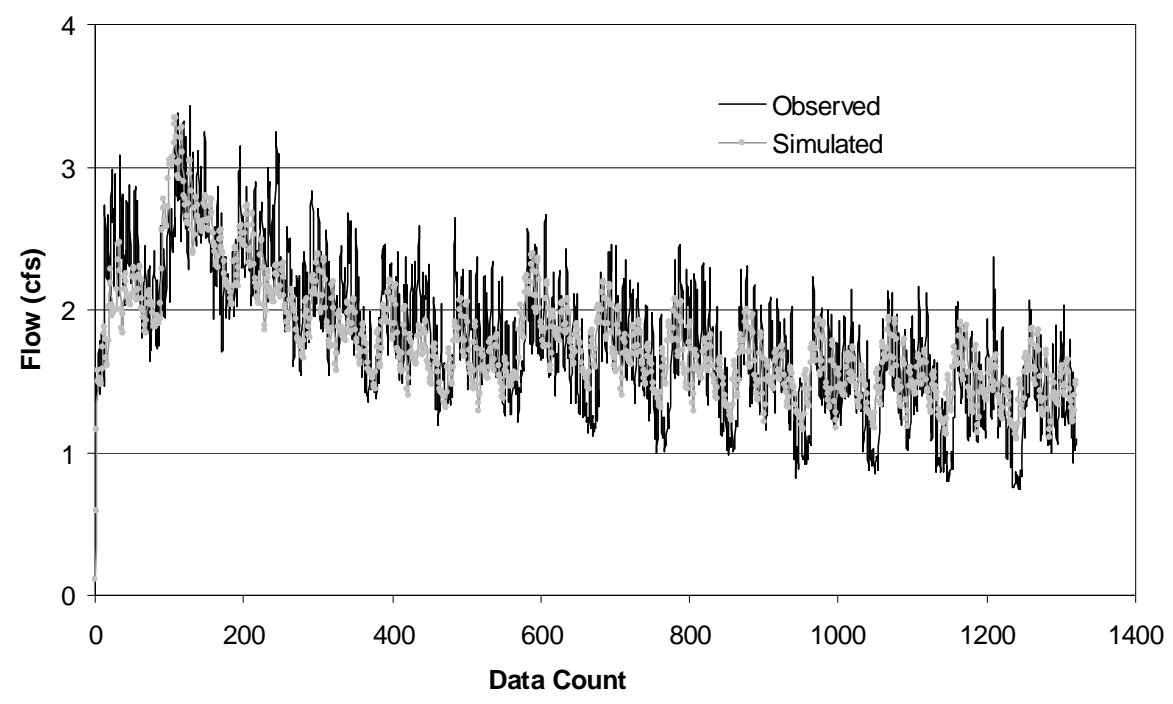

Figure 2: Plot of Observed versus Simulated RDII Flow. 
The calibration problem was solved with GA optimization. A population size of 100 was used and the maximum generation was set to fifty. A time series plot that compares the decomposed RDII flow with the RDII flow simulated by the calibrated model is given in Figure 2. As shown in the figure, excellent agreement is achieved. The optimal design model was applied to identify cost-effective design improvements so that capacity of the existing pipes is not exceeded. Design results that show the costs incurred by the best ten proposed solutions are also given in Figure 3.

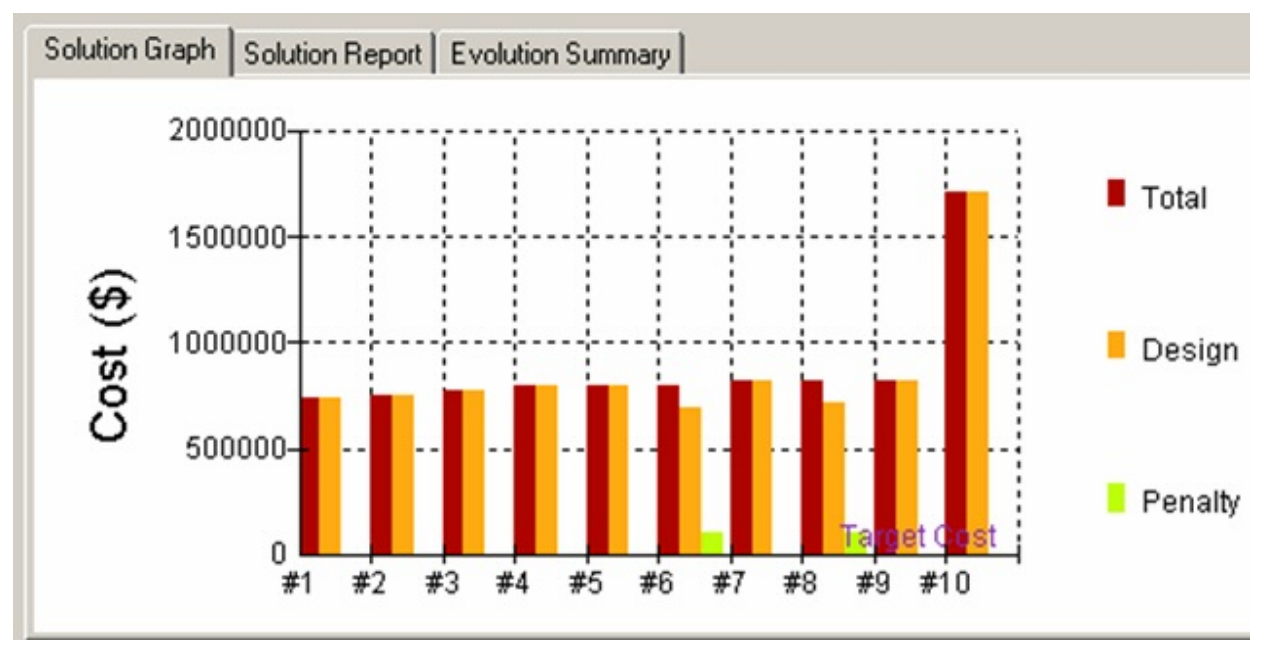

Figure 3: Plot of the Costs Incurred by the Top Ten Best Design Solutions.

\section{Conclusions}

Rainfall driven infiltration and inflow (RDII) is a major source of sanitary sewer overflows (SSOs). Computer based simulation models are powerful tools for evaluating sewer collection system performance, identifying system deficiencies, and assessing the efficacy of the proposed design improvement alternatives. In this paper, we described a rigorous and efficient three-step optimization methodology for use in solving the sewer overflow problem. The proposed approach performs rigorous QA/QC of rainfall and meter data and decomposes flow data into dry-weather flow (DWF) and wet-weather flow (RDII) components. The DWF component is further analyzed to construct a DWF pattern. This pattern is then assigned to the source nodes that contribute flow to the meter location, proportional to sewershed areas or based on other user-defined criteria. Next, using genetic algorithm optimization, the optimal RTK parameters that best match the RDII time series generated by decomposing the measured flow data are determined. The resulting inflow hydrograph (RDII + DWF) is then used by the sewer simulation model to carry out detailed dynamic flow routing through the sewer system and evaluate system response to support development of an optimal capital improvement program. In the final step, the calibrated model is then used to design cost-effective remedial solutions for existing SSO problems. The design model determines the set of design parameters that best meets desired system performance criteria at minimum cost. Design parameters can include any combination of pipe size, storage, slope, and pumping. System performance criteria include explicit constraints on the maximum allowable depth to diameter ratio to 
avoid SSOs, minimum and maximum pipe velocities, and maximum head loss for force mains. The proposed wet-weather flow decomposition, optimal calibration, and optimal design models will enable much more complex SSO problems to be solved than was previously possible.

\section{References}

Bennett, D. et al. (1999). Using flow prediction technologies to control sanitary sewer overflows. Water Environment Research Foundation (WERF), Project 97-CTS-8, Alexandria, VA.

Boulos, P.F. (2005). InfoSWMM Suite - Users guide. MWH Soft, Inc., Broomfield, CO.

Boulos, P.F. (2006). $\mathrm{H}_{2}$ OMAP SWMM Suite - Users guide. MWH Soft, Inc., Broomfield, $\mathrm{CO}$.

Cieniawski, S.E., Eheart, J.W., and Ranjithan, S. (1995). "Using genetic algorithms to solve a multiobjective groundwater monitoring problem." Journal of Water Resources Research, 31(2), 399-409.

Holland, J.H. (1975). Adaptation in natural and artificial systems. University of Michigan Press, Ann Arbor, MI.

Nicklow, J.W., Boulos, P.F., and Muleta, M.K. (2004) Comprehensive sewer collection systems analysis handbook for engineers and planners. MWH Soft, Inc. Publ., Broomfield, CO.

Nicklow, J.W., Boulos, P.F., and Muleta, M.K. (2006). Comprehensive urban hydrologic modeling handbook for engineers and planners. MWH Soft, Inc. Publ., Broomfield, CO.

Rossman, L.A. (2005). Stormwater management model SWMM - Users manual Version 5. U.S. Environmental Protection Agency, National Risk Management Research Laboratory, Water Supply and Water Resources Division, Cincinnati, $\mathrm{OH}$.

U.S. Environmental Protection Agency (2004). Report to Congress: Impacts and control of CSOs and SSOs. Report No. EPA/833/R-04-001, EPA, Washington, D.C. 